\title{
Predictores familiares de la violencia filio-parental: el papel de la disciplina familiar
}

\author{
Izaskun Ibabe
}

Universidad del País Vasco

\begin{abstract}
Resumen: La violencia filio-parental es un problema social cualitativamente diferente a otros tipos de violencia familiar, porque los adolescentes dirigen la violencia hacia quienes debieran representar la autoridad y proporcionarles bienestar. El objetivo principal de este estudio era analizar el papel de las relaciones paternofiliales y la disciplina familiar en el desarrollo de conductas violentas y prosociales de los adolescentes hacia sus padres. En el estudio participaron 585 hijos/as (48\% varones) entre 12 y 18 años procedentes de 8 centros escolares de la Comunidad Autónoma del País Vasco. Los resultados indican que las relaciones familiares basadas en el afecto y la comunicación son las que favorecen las conductas prosociales de los hijos, y reducen las conductas violentas de éstos en el hogar. Por el contrario, las estrategias de disciplina parental coercitivas y las estrategias parcialmente coercitivas (supervisión y coste de respuesta) se asocian a un mayor nivel de violencia física y psicológica de los hijos adolescentes hacia sus padres. Finalmente, se discuten las implicaciones de estos resultados en relación a la educación parental.

Palabras clave: Violencia filio-parental; estilos de socialización parental; disciplina familiar; violencia marital.
\end{abstract}

\section{Introducción}

La adolescencia constituye un periodo evolutivo caracterizado por numerosos cambios biológicos, psicológicos y sociales, siendo habitual en esta etapa los conflictos familiares entre progenitores e hijos/as. Los hijos e hijas reclaman sobre todo autonomía y una relación más igualitaria (Rodrigo, García, Márquez y Triana, 2005), y esto crea situaciones de conflicto familiar con más frecuencia que en las etapas evolutivas anteriores. En estas situaciones puede haber tanto agresión verbal como física de hijas o hijos hacia las madres y padres de diferentes niveles de severidad. En el contexto español, las conductas violentas de hijos/as adolescentes a padres y madres (violencia filio-parental) parece que han ido en aumento desde el año 2006, si tenemos en cuenta el número de denuncias de padres agredidos por sus hijos según los datos aportados por la Fiscalía General del Estado (Matey, 2011). Así, en 2007 fueron 2.683 padres los que denunciaron a sus hijos mientras que en 2010 sumaron 8.000 casos. También cabe destacar que este crecimiento en el número de casos en los últimos años se ha constatado también en otros países como Australia (p.e, NTV, 2012) o Reino Unido (Condry, 2011). Aunque es posible que este aumento sea reflejo del afloramiento de un problema oculto en el seno de la familia que ya existía anteriormente.

En Norte América los índices de violencia de hijos a padres se encuentran en un rango entre el 7\% y 29\%, mientras que en Canadá y Francia los índices son ligeramente inferiores (ver las revisiones de Gallagher, 2008, y Kennair y Me-

* Dirección para correspondencia [Correspondence address]:

Izaskun Ibabe. Dpto. de Psicología Social y Metodología de las Ciencias del Comportamiento. Facultad de Psicología, Universidad del País Vasco, Avda. de Tolosa, 70, 20018-Donostia-San Sebastián (España).

E-mail: izaskun.ibabe@,ehu.es
Title: Family predictors of child-to-parent violence: the role of family discipline.

Abstract: Child-to-parent violence is a social problem that is qualitatively different from other types of family violence, since adolescents direct their violence toward those who should represent authority and provide for their welfare. The main goal of this study was to analyze the role of family relationships and family discipline on violent and prosocial behavior by adolescents toward their parents. Participants were 585 children aged between 12 and 18 from 8 schools in the Autonomous Community of the Basque Country (Spain). Results show that family relationships based on affect and communication are those that promote prosocial behaviors by children and reduce their violent behaviors at home. However, power-assertive parental discipline strategies and partially punitive strategies (monitoring and penalty) were associated to more physical and psychological violence by adolescents toward their parents. Finally, implications of these findings for parenting education are discussed.

Key words: Child-to-parent violence; parenting styles; family discipline; marital violence.

llor, 2007). En el contexto español, en varios estudios sobre la población general se ha comprobado que entre el 3\% y el $21 \%$ de los progenitores han sufrido en alguna ocasión episodios de agresión física por parte de sus hijos adolescentes (Calvete, Orue y Sampedro, 2011; Gámez-Guadix, Jaureguizar, Almendros y Carrobles, 2012; Ibabe y Jaureguizar, 2011; Jaureguizar e Ibabe, 2012). El estudio de la prevalencia y los factores de riesgo de la violencia de hijos a padres ha recibido una atención creciente en la última década tanto a nivel internacional (Bobic, 2004; Walsh y Krienert, 2007), como a nivel nacional (p.e., Calvete et al., 2011; Gámez-Guadix et al., 2012), sin embargo, todavía es escasa la evidencia empírica disponible en torno a la relación entre la violencia de hijos a padres y las variables familiares.

La familia sigue siendo el más importante agente de socialización, a pesar de todos los cambios experimentados en las últimas décadas en cuanto a su estructura o el cambio en los roles de los padres y madres. La comunidad científica se ha interesado por el estudio de las posibles repercusiones que dicha transformación puede acarrear para el desarrollo psicológico infantil (Antolín, Oliva y Arranz, 2009). Los cambios en la estructura familiar se han asociado tradicionalmente a la presencia de problemas de conducta y/o conductas delictivas así como a problemas en las relaciones paternofiliales (Hetherington y Henderson, 1997). En un estudio longitudinal realizado por Pagani, Larocque, Vitaro y Tremblay (2003) en el que se analizó el desarrollo de conductas violentas ejercidas por 778 hijos/as hacia sus padres a lo largo de toda su infancia hasta la adolescencia, se halló que los cambios en el subsistema marital (divorcio o nuevo matrimonio) suponían un factor de riesgo de agresiones físicas contra las madres en comparación con las familias que se mantenían intactas en todo el período. Sin embargo, Kennair y Mellor (2007) en su revisión señalan que la violencia filio- 
parental es más habitual en las familias monoparentales que en las familias tradicionales.

\section{Clima familiar}

El clima familiar positivo hace referencia a las prácticas de socialización de la familia que fomentan la confianza, la autonomía, la iniciativa y los lazos afectivos para el desarrollo de hijas e hijos estables y competentes (Werner, 1989). Hay evidencia empírica de que el clima familiar positivo es un factor protector de la violencia filio-parental (VFP) evaluado a través de la cohesión familiar, relaciones paternofiliales positivas y conductas prosociales. Las relaciones positivas entre padres/madres e hijos/as se han asociado con un menor riesgo de abuso verbal o físico de las y los adolescentes hacia sus madres y/o padres (Jaureguizar e Ibabe, 2012; Pagani et al., 2003) y con un mayor desarrollo de conductas prosociales en el ámbito familiar (Jaureguizar e Ibabe, 2012; Romano, Tremblay, Boulerice y Swisher, 2005). Los resultados del estudio de Jaureguizar e Ibabe (2012) ponen de relieve el impacto de la cohesión familiar y la expresión de sentimientos positivos en el desarrollo de conductas prosociales entre los miembros de la familia, y en la reducción de conductas violentas hacia los progenitores. Además, en este estudio se encontró una relación inversa entre las conductas violentas y conductas prosociales de las hijas e hijos hacia los progenitores. Parece que las relaciones padres-hijos, basadas en el afecto y la comunicación, sirven para prevenir la violencia filio-parental y las conductas antisociales en general.

Por el contrario, el clima familiar negativo es uno de los factores de riesgo más conocidos de la violencia filioparental y está caracterizado por un elevado nivel de conflicto familiar, bajo nivel de cohesión y presencia de violencia familiar (violencia marital y violencia de padres a hijos). Los adolescentes agresivos describen a sus progenitores como personas que les rechazan, son sobreprotectoras y/o menos cálidas emocionalmente (Aluja, Del Barrio y García, 2005). También se ha comprobado empíricamente que el conflicto familiar es un predictor tanto de las conductas violentas hacia las figuras de autoridad como de las conductas antisociales (Jaureguizar e Ibabe, 2012). En esta misma línea, las hijas e hijos que viven en familias con más aspectos disfuncionales presentan menores niveles de conductas pro-sociales (Romano et al., 2005). Además, los resultados de diversos estudios apoyan la hipótesis de la bidireccionalidad de la violencia familiar: los adolescentes que experimentan directamente violencia familiar o son testigos de violencia marital entre sus padres, son más propensos a presentar comportamientos agresivos hacia sus padres (Calvete et al., 2011; Gámez-Guadix y Calvete, 2012; Ibabe y Jaureguizar, 2011; Kennedy, Edmonds, Dann y Burnett, 2010; Ulman y Straus, 2003). La explicación de tal bidireccionalidad puede residir en el aprendizaje de modelos de relación basados en la violencia, a través del cual los niños interiorizan que la única forma de resolver los conflictos es mediante el uso de tácticas violentas (p.e., Mitchell y Finkelhor, 2001).

\section{Estilo parental}

El constructo de estilo parental se utiliza para recoger las variaciones normales en los intentos de los padres para controlar y socializar a sus hijas e hijos (Baumrind, 1991), que incluye cuatro estilos parentales: autoritario -elevados niveles de control y escaso afecto-, democrático-elevados niveles de control y afecto-, indulgente -bajo control y elevado afecto- y negligente -bajo control y bajo afecto-. Esta categorización está basada en dos ejes: afecto-comunicación y exigenciacontrol (Maccoby y Martin, 1983).

De acuerdo con la literatura científica, el estilo democrático -al menos en las culturas occidentales- se relaciona con niveles más elevados de ajuste, madurez psicosocial, competencia psicosocial, autoestima y éxito académico (p.e., Musitu, Buelga, Lila y Cava, 2001) mientras que los estilos autoritario, indulgente y negligente han sido generalmente relacionados con consecuencias negativas para el menor, como el estrés emocional, los síntomas somáticos o la conducta antisocial (Lamborn, Mounts, Steinberg y Dornbusch, 1991). Sin embargo, en el contexto español y en algunos países latinoamericanos existen estudios que indican que los adolescentes de familias indulgentes obtienen puntuaciones iguales o mayores en diferentes criterios sobre su ajuste psicológico que los adolescentes de familias que aplican el estilo democrático (p.e., García y García, 2010; Musitu y García, 2004) y un menor nivel de conductas violentas hacia sus padres y/o madres (Casteñeda, Garrido-Fernández y Lanzarote, 2011; Gámez-Guadix et al., 2012).

En conclusión, en nuestro contexto cultural la falta de afecto e implicación paterna en la educación de los hijos (estilos autoritario y negligente) estarían relacionados con la manifestación de agresividad dirigida hacia los progenitores, mientras que los estilos parentales basados en el afecto (estilos indulgentes y democráticos) se relacionarían con un menor nivel de agresividad verbal y física de los hijos hacia los padres. Sin embargo, la relación entre estilo parental y violencia filio-parental podría estar modulada o mediada por el contexto cultural. Esto significa que las consecuencias de los diferentes procesos de socialización familiar pueden diferir de un contexto cultural a otro (Calvete, Gámez-Guadix y Orue, 2010; Gámez-Guadix et al., 2012).

\section{Disciplina familiar}

El proceso de socialización de los hijos por parte de los padres es complejo e incluye tanto estrategias de control como demostraciones de afecto (Straus, 1964). Con el fin de favorecer o prevenir determinados comportamientos en el hijo o hija los padres y madres aplican determinadas estrategias de disciplina. En el ámbito del desarrollo del niño la disciplina familiar se refiere a los métodos para moldear el carácter y enseñar a auto-controlarse y fomentar las conductas aceptables (Papalia, Olds y Feldman, 2007). Las estrategias de disciplina familiar se han clasificado en coercitivas, inductivas e indiferentes (Musitu, Román y Gracia, 1988). 
Las estrategias coercitivas implican el seguimiento de un comportamiento inadecuado de un niño con una consecuencia negativa (amenazas o retirada de privilegios) sin explicación o justificación. Por su parte, las estrategias inductivas implican poner límites, mostrar las consecuencias lógicas de su conducta a través de la reflexión, negociación, razonamiento y explicación (Holden, 2002). Por último, las estrategias indiferentes incluyen aspectos como indiferencia, permisividad y pasividad. Además, Straus y Fauchier (2007) clasificaron las estrategias de disciplina parental según el nivel de coerción: coercitivas (castigo físico y psicológico), parcialmente coercitivas (retirada de privilegios y compensación) y no coercitivas (disciplina inductiva). Los mismos autores relacionaron el estilo educativo parental con la disciplina familiar. Así, caracterizaron el estilo democrático con un nivel medio de coerción, elevado nivel de afectividad y razonamiento, mientras que el estilo indulgente se caracteriza por un bajo nivel de coerción, elevado nivel de afectividad y razonamiento. Un elemento clave de la disciplina inductiva es que está basada en las cogniciones de lo que está bien y de lo que está mal, y especialmente en el aprendizaje de los niños de su comportamiento inadecuado sobre otros (Hoffman, 1983).

En diversos estudios se ha comprobado que las prácticas de disciplina parental inadecuadas, como los castigos físicos tienen consecuencias negativas a largo plazo en el ajuste psicológico del hijo o hija (p.e., Gámez-Guadix, Straus, Carrobles, Muñoz-Rivas y Almendro, 2010; Musitu y García, 2004), así como en las conductas violentas de hijos a padres (Brezina, 1999; Straus y Stewart, 1999). Asimismo, el bajo nivel de supervisión es un factor de riesgo para el desarrollo de conductas antisociales en la adolescencia (Loeber, y Stouthamer-Loeber, 1986; Yoshikawa, 1994), pero la disciplina parental basada en el excesivo control autoritario estaría asociado a la violencia en el hogar (Beyers y Goossens, 1999; Weiss y Schwarz, 1996). A pesar de todo lo anterior, la dimensión control/coerción ha planteado desacuerdos en su definición entre los investigadores así como diferencias en su evaluación, acarreando problemas para concluir los efectos que presenta esta dimensión en el ajuste del menor (Lorence, 2007). También hay autores que cuestionan la importancia del control parental para la prevención de los problemas comportamentales en niños y adolescentes (Oliva, 2006).

\section{Objetivos e hipótesis del estudio}

Apenas hay estudios sobre la relación entre las estrategias de disciplina inductiva o estrategias de disciplina parcialmente coercitivas (coste de respuesta y supervisión) y las conductas violentas, por lo que el presente estudio tenía por objetivo determinar el papel que juegan la calidad de las relaciones familiares y las estrategias de disciplina familiar según el nivel de coerción en el desarrollo de las manifestaciones de violencia y conductas prosociales de los adolescentes hacia sus padres. De forma complementaria también se pretendían estudiar las posibles diferencias que pudieran existir entre diferentes tipos de familia (según la estructura familiar y el con- texto cultural) en relación a la VFP. Para ello se utilizó un modelo SEM (Structural Equation Modeling) para evaluar la capacidad predictiva de las variables familiares en la VFP. Las hipótesis propuestas fueron las siguientes:

a) La violencia marital física será un predictor válido de la violencia física que los hijos ejercen hacia sus padres (Kennedy et al., 2010; Ulman y Straus, 2003) así como del clima familiar negativo ${ }^{1}$ (Gallagher, 2008; Pagani et al., 2003).

b) Las estrategias altamente coercitivas de disciplina familiar (Straus y Stewart, 1999) y las moderadamente coercitivas se asociarán positivamente con la violencia física de los hijos hacia los padres, mientras que las estrategias no coercitivas (disciplina inductiva) lo harán inversamente o no se relacionarán (Calvete et al., 2011).

c) Las estrategias de disciplina coercitivas se aplicarán con mayor frecuencia en las familias inmigrantes que en las familias autóctonas.

d) La tasa de violencia filio-parental física será menor en las familias nucleares que en las familias monoparentales con la madre (Kennair y Mellor, 2007), en familias reconstituidas, o familias de otro tipo (monoparentalpadre, familia extensa o familia de acogida).

e) Las conductas prosociales de hijos a padres se relacionarán con un clima familiar positivo (Jaureguizar e Ibabe, 2012; Romano et al., 2005) y la disciplina inductiva.

\section{Método}

\section{Participantes}

En este estudio participaron un total de 585 adolescentes de la Comunidad Autónoma Vasca (España), de edades comprendidas entre 12 y 18 años $(M=14.76 D T=1.6)$ de ambos sexos (48\% varones) procedentes de 8 centros escolares. El 43\% de los participantes procedían de centros públicos mientras que el resto pertenecían a centros concertados. El 75\% de ellos vivía en familias nucleares, el 14\% en familias monoparentales con la madre, el $7 \%$ en familias reconstituidas y el $4 \%$ en otro tipo de familias (monoparentalpadre, familia extensa, familias de acogida y homoparental). Además, el 23\% de los participantes pertenecían a familias inmigrantes.

\section{Instrumentos}

Variables socio-demográficas. En un cuestionario desarrollado ad hoc se recogió la información relativa a los datos sociodemográficos de los participantes, y entre las variables recogidas se encuentran sexo, edad, país de origen, estructura familiar, nivel educativo y profesión de los padres.

Violencia filio-parental. Se utilizaron las Escalas Tácticas para Conflictos Hijo-Padres (CTS1; Straus, Hamby, Finkelhor, Moore y Runyan, 1998). Este instrumento contiene 16

${ }^{1}$ Clima familiar negativo: Baja cohesión familiar y elevada conflictividad. 
ítems pertenecientes a cuatro dimensiones: razonamiento, agresión psicológica (p. e., “¿Has gritado a tu padre/tu madre?”), violencia física leve y violencia física grave. Cada uno de los ítems había de ser respondido dos veces, uno respecto a la madre y otro respecto al padre. Los participantes respondieron tomando como referencia el último año e informando sobre la frecuencia en una escala con los siguientes valores: 0 (Nunca), 1 (Una vez), 2 (A veces), 3 (Con bastante frecuencia) y 4 (Con mucha frecuencia). La validez de las escalas CTS ha sido analizada en numerosos estudios (p.e., Straus y Douglas, 2004; Straus, Hamby, Boney-McCoy y Sugarman, 1996). Los resultados de la fiabilidad de este estudio son bastante aceptables tanto para la globalidad $(\alpha=.83)$ como para cada dimensión (razonamiento $\alpha=.64$; agresión psicológica $\alpha=.85$; violencia física leve $\alpha=.76$; violencia física grave $\alpha$ $=.79)$.

Violencia Marital. Se utilizaron las Escalas Tácticas Revisadas (CTS2; Straus, Hamby, Boney-Mccoy y Sugarman, 1996). Este instrumento evalúa la violencia entre los padres y tiene 6 ítems bidireccionales (de padre a madre $v$ s. de madre a padre) sobre violencia física (p.e., "¿'Tu padre empujó o apartó de un empujón a tu madre?”), y está basada en la Escala de Relaciones Conductuales. Los adolescentes informaron sobre la frecuencia en una escala de 5 categorías desde 1 (Nunca) hasta 5 (Con mucha frecuencia) con la que sus padres llevaron a cabo diferentes comportamientos violentos entre ellos en el último año. Para analizar su validez de constructo, se efectuó un análisis de componentes principales y rotación varimax de la escala en esta muestra. Dos factores alcanzaron a explicar el $66 \%$ de la varianza total y con autovalores superiores a 1 . El primer factor (autovalor $=5.23$ ) agrupaba los ítems relacionados con las agresiones de padre a madre y el segundo factor (autovalor $=2.73$ ) los relacionados con las agresiones de madre a padre. Todos los ítems tenían cargas factoriales por encima de .60. En este estudio los coeficientes alfa eran aceptables (padre-madre $\alpha=.92$ y madre-padre $\alpha=.79$ ).

Disciplina familiar. El instrumento utilizado para evaluar las estrategias de disciplina familiar fue el Inventario de Dimensiones de Disciplina (DDI-C; Straus y Fauchier, 2007; adaptación española de Calvete et al., 2010). Este inventario consta de 26 ítems y evalúa la disciplina familiar que perciben los hijos tanto en su relación con el padre como con la madre (p.e., "Obligar a hacer tareas extra como castigo"). Este instrumento evalúa cuatro dimensiones generales: Castigo (físico-psicológico), Coste de respuesta (retirada de privilegios y compensación), Supervisión (ignorar y controlar) y Disciplina inductiva (distracción ${ }^{2}$, explicación y recompensa). Los ítems plantean diferentes situaciones propias de la vida y educación familiar a las que los jóvenes deben contestar en una escala de 5 puntos de tipo Likert de 0 (Nunca) a 4 (Casi siempre). En este estudio se obtuvo una excelente con-

\footnotetext{
${ }^{2}$ Distracción: Los padres ofrecen a los hijos hacer algo diferente en lugar de lo que está haciendo mal o les envían a otra habitación por un periodo determinado.
}

sistencia interna para este inventario $(\alpha=.92)$. Los coeficientes de las subescalas eran aceptables: Castigo $(\alpha=.86)$, Coste de respuesta $(\alpha=.87)$, Supervisión $(\alpha=.77)$ y Disciplina inductiva $(\alpha=.81)$.

Clima familiar. Se aplicaron tres subescalas de la Escala de Clima Social Familiar (FES; Moos y Moos, 1981; adaptación española de TEA ediciones, 1984) con un total de 27 ítems del tipo verdadero-falso: Cohesión (grado en que los miembros de la familia están compenetrados, se ayudan y se apoyan entre sî), Conflicto (grado en que se expresan libre y abiertamente la cólera, agresividad y conflicto entre los miembros de la familia) y Organización (importancia que se da a una clara organización y estructura al planificar las actividades y responsabilidades de la familia). Un ejemplo de estos ítems sería: "En mi familia hay un fuerte sentimiento de unión". El coeficiente alfa global $(\alpha=.81)$ y la subescala Cohesión $(\alpha=.76)$ eran aceptables mientras dos subescalas presentaban valores por debajo del nivel deseable $(\alpha>.70)$ : Conflicto $(\alpha=.61)$ y Organización $(\alpha=.52)$.

Conductas prosociales en la familia. En este contexto, la conducta prosocial se define como cualquier comportamiento que beneficia a otros o que tiene consecuencias sociales positivas e incluye conductas de ayuda, cooperación y solidaridad (Moñivas, 1996). Para evaluar esta variable se utilizó un instrumento adaptado de la Escala de Conductas hacia las Figuras de Autoridad (Jaureguizar e Ibabe, 2012) con propiedades psicométricas aceptables. A la subescala original de conductas prosociales hacia los padres se añadieron 4 ítems más, siendo 7 ítems en total los que evalúan las conductas y actitudes prosociales en el ámbito familiar de hijos hacia padres y hermanos (p.e., "En las discusiones que tenemos en casa, mantengo la calma e intento solucionar los conflictos hablando con ellos"). Los adolescentes tienen que valorar la frecuencia con la que realizan determinadas conductas prosociales en el ámbito familiar en una escala de tipo Likert de 0 (Nunca) a 4 (Muchas veces) puntos. El análisis factorial de componentes principales y rotación Varimax arrojó una estructura de dos factores (conductas prosociales hacia los progenitores y hacia los hermanos/as) cada uno con valores propios superiores a 1 , que en conjunto explican $57 \%$ de la varianza total. En este estudio la consistencia interna global fue aceptable $(\alpha=.75$ ), así como la de las subescalas (conducta prosocial padres $\alpha=.85$; conducta prosocial hermanos $\alpha=.67)$.

\section{Procedimiento}

La selección de la muestra de los hijos adolescentes se realizó a través de un muestreo por conglomerados. Una vez conseguidas las autorizaciones pertinentes de los centros, se procedió a seleccionar el modelo lingüístico (bilingüe $v s$. monolingüe), y el nivel de estudios de los participantes dentro de cada centro escolar, para conseguir una muestra equilibrada y representativa. A las direcciones de los centros educativos que decidieron participar en la investigación se les proporcionó la información precisa acerca de la investi- 
gación y se pactaron las condiciones de envío del protocolo de consentimiento informado a los padres de cada estudiante. El alumnado participante entregó el consentimiento firmado por sus progenitores a sus respectivos maestros/as tutores, y posteriormente fueron informados de los objetivos del estudio, del carácter anónimo y confidencial de la información aportada, así como de la voluntariedad de su participación. Las instrucciones para la recogida de datos estaban estandarizadas y descritas paso a paso. Se contrabalanceó el orden de presentación de los instrumentos de medida. La recogida de datos se realizó durante el año 2011 en horario regular de clase estando presente el personal adscrito a esta investigación. El tiempo de administración de los cuestionarios e instrumentos de evaluación fue de 45 minutos aproximadamente. Una vez analizados los datos, se ofreció una charla informativa a las direcciones de los centros participantes en el estudio sobre los factores asociados a la violencia intra-familiar.

\section{Análisis de datos}

Los análisis de datos se realizaron con el programa SPSS versión 20. Para obtener la prevalencia de la VFP y la violencia marital, se dicotomizaron las variables teniendo en cuenta el formato de respuesta $(0=$ Nunca, $1=$ Una vez, $2=$ $A$ veces, $3=$ Con bastante frecuencia, y $4=$ Con mucha frecuencia $)$, y los participantes que elegían la opción 1 o superior se consideró que ejercían o habían ejercido algún tipo de violencia hacia sus padres en el último año (entre 2010 y 2011). Esto significa que los porcentajes que se presentan incluyen también la violencia ejercida de forma ocasional o esporádica en el último año. En segundo lugar, se realizaron diversos análisis Chi-cuadrado relacionados con el tipo de estructura familiar (nuclear, monoparental-madre, reconstituida y otros) para la violencia filio-parental. Las familias monoparentales con el padre se incluyeron en otro tipo de familia, debido al reducido número de este tipo de estructura familiar $(n=8$; $1 \%$ y por las características diferenciales que pudiera tener respecto a la familia monoparental con la madre. En tercer lugar, se efectuaron los análisis de correlación de Pearson para comprobar la relación entre la VFP y las conductas prosociales de los hijos con el resto de variables estudiadas en el contexto familiar (violencia marital, clima familiar y estrategias de disciplina).

Además, se utilizó el programa EQS 6.1 (Structural Equation Program) para evaluar si el modelo estructural de la violencia filio-parental era adecuado. El objetivo del modelo era estudiar el papel de las variables familiares en la violencia filio-parental. Las variables latentes que se incluyeron en el modelo fueron: Violencia Filio-Parental (indicadores: violencia física y psicológica), Conductas Prosociales (indicadores: padres y hermanos), Clima Familiar Positivo (indicadores: cohesión, bajo conflicto y organización), Disciplina Coercitiva (indicadores: castigo, coste de respuesta y supervisión) y Disciplina Positiva (indicadores: distracción, explicación y recompensa). Se permitieron correlaciones signifi- cativas entre los residuales de Disciplina Coercitiva, Disciplina Positiva y Violencia Filio-Parental por tratarse de un estudio de corte transversal. El Clima Familiar Positivo predecía la Disciplina Coercitiva, la Conducta Prosocial y la Violencia Filio-Parental. También se incluyeron en el modelo dos variables observables independientes: violencia marital e inmigrante. La violencia marital física se incluyó como predictor del Clima Familiar Positivo y de la violencia física (de hijos a padres). No se descarta la posibilidad de que la condición de inmigrante pueda predecir la violencia filioparental y se examinan los resultados de la prueba del multiplicador de Lagrange (Chou y Bentler, 1990) para evaluar la posibilidad de añadir otros parámetros al modelo, y mejorar así el ajuste y/o la capacidad explicativa. Los índices prácticos de ajuste utilizados fueron IFI, CFI y NNFI, y se esperaban valores mayores que .90 para estos indicadores (Bentler, 2006). El índice RMSEA se utiliza para medir el error razonable de aproximación en términos de bondad de ajuste, requiriendo un valor inferior a $.06(\mathrm{Hu}$ y Bentler, 1999). El análisis se llevó a cabo con la información completa utilizando el método de máxima verosimilitud (p.e., Arbuckle, 1996). Como el coeficiente de curtosis de Yuan, Lambert y Fouladi (2004) normalizado era elevado (78.32) se aplicó también el método robusto tanto para el modelo de medida como para el modelo estructural de Yuan-Bentler (2000). Como era esperable, en algunas distribuciones univariadas se detectó una marcada falta de normalidad: violencia física (asimetría $=6.91$; curtosis $=58.55)$ y violencia marital (asimetría $=6.50 ;$ curtosis $=53.44)$. En dichas variables la mayoría de los adolescentes no mostraba ninguna tendencia a la violencia o el nivel era muy bajo y lo mismo ocurría con la violencia marital (violencia física entre padres). En la sección de resultados se presentan los índices de ajuste basados en la distribución normal y en la estadística robusta.

\section{Resultados}

\section{Prevalencia de la violencia filio-parental y violencia marital}

El 5\% de los adolescentes manifestaron haber ejercido algún tipo de violencia física grave hacia alguno de sus padres en alguna ocasión, el 11\% violencia física leve y el 13\% ejercía alguno de los dos tipos de violencia. La prevalencia del abuso físico considerando las conductas específicas variaba entre el 1\% ("Dar una paliza") y el 9\% ("Empujar o apartar de un empujón"). No se hallaron diferencias significativas para la perpetración de agresión física (leve, grave y global) en función del sexo de los progenitores y el sexo de los hijos, ni tampoco al analizar las conductas específicas de cada tipo de violencia. Además, se encontró que en las familias nucleares la tasa de VFP física $(10 \%)$ era menor que en las familias monoparentales con la madre $(21 \%) \chi^{2}(1, N=$ $481)=4.94, p=.026, r=.10$, en familias reconstituidas $(22 \%), \chi^{2}(1, N=473)=5.60, p=.018, r=.11$, o que en $\mathrm{fa}-$ 
milias de otro tipo, $\chi^{2}(1, N=450)=10.46, p<.001, r=$ .15 .

El 88\% de la muestra declaró haber ejercido alguna conducta de violencia psicológica hacia sus padres. La prevalencia del abuso psicológico oscilaba entre el 16\% ("Amenazar con golpearles") y el $84 \%$ ("Gritarles"). Este tipo de abuso era algo más frecuente hacia las madres (84\%) que hacia los padres $(81 \%), \chi^{2}(1, N=545)=223.91, p<.01, r=.64$. Por otro lado, las hijas ejercían ligeramente más conductas de violencia psicológica hacia las madres $(88 \%)$ en comparación con los hijos (82\%), $\chi^{2}(1, N=566)=5.20, p=.02, r=.09$.

Respecto a la violencia marital, según los autoinformes de los hijos e hijas, en el 11\% de las familias había algún tipo de violencia física entre los progenitores: violencia unidireccional $(7 \%)$ y violencia recíproca $(4 \%)$. Los porcentajes variaban dependiendo de la conducta violenta: "dar una paliza" ( $1 \%$ padre-madre y $0.4 \%$ madre-padre) y "empujar o apartar de un empujón" ( $6 \%$ padre-madre y $7 \%$ madre-padre). El porcentaje de violencia unidireccional de madre a padre $(5 \%)$ era ligeramente superior al de padre a madre $(2 \%)$, $\chi^{2}(1, N=43)=6.72, p<.01$

\section{Relación entre la VFP y características familiares}

De acuerdo con la Tabla 1, podemos afirmar que la VFP, tanto física como psicológica, se asocia significativamente a un mayor nivel de violencia entre los padres $(r=.45$ y $r=$ $.24)$, conflicto familiar $(r=.20 \mathrm{y} r=.48)$, y a una mayor frecuencia de aplicación de estrategias coercitivas como son el castigo $(r=.27$ y $r=.58)$, coste de respuesta $(r=.10$ y $r=$ .27) y supervisión $(r=.18$ y $r=.42)$, así como a una menor cohesión $(r=-.21$ y $r=-.42)$, y organización familiar $(r=-$ .14 y $r=-.27)$. Inversamente a lo hallado para las conductas violentas, las conductas prosociales de los hijos hacia los padres se relacionaban con un menor nivel de violencia marital $(r=-.12)$, conflicto familiar $(r=-.41)$, aplicación de estrategias coercitivas por parte de sus progenitores (castigos $r=$ .36 y supervisión $r=-.21$ ), así como con un mejor clima familiar (cohesión $r=.47$ y organización $r=.34$ ). Los resultados de las conductas prosociales hacia los hermanos seguía el mismo patrón de resultados. Sin embargo, algunas estrategias de disciplina positiva (distracción $r=-.11$; recompensa $r$ $=-.11$ ) se asociaban débilmente con un menor nivel de violencia marital. También cabe destacar que la disciplina positiva no se relaciona ni con la VFP ni con las conductas prosociales.

Tabla 1. Medias, desviaciones típicas y correlaciones entre la VFP y variables asociadas al contexto familiar.

\begin{tabular}{|c|c|c|c|c|c|c|c|c|c|c|c|c|c|c|c|}
\hline & $M$ & DT & 1 & 2 & 3 & 4 & 5 & 6 & 7 & 8 & 9 & 10 & 11 & 12 & 13 \\
\hline \multicolumn{16}{|l|}{ Violencia filio-parental } \\
\hline 1. Violencia física & .04 & .18 & - & & & & & & & & & & & & \\
\hline 2. Violencia psicológica & .62 & .52 & $.38^{* *}$ & - & & & & & & & & & & & \\
\hline \multicolumn{16}{|l|}{ Conductas prosociales } \\
\hline 3. Hacia padres & 2.72 & .70 & $-.20^{* *}$ & $-.40^{* *}$ & - & & & & & & & & & & \\
\hline 4. Hacia hermanos & 3.01 & 1.06 & $-.13^{* *}$ & $-.17^{* *}$ & $.43^{* *}$ & - & & & & & & & & & \\
\hline \multicolumn{16}{|l|}{ Clima familiar } \\
\hline 5. Violencia marital ${ }^{1}$ & .05 & .19 & $.45^{* *}$ & $.24^{* *}$ & $-.12^{* *}$ & $-.15^{* *}$ & - & & & & & & & & \\
\hline 6. Conflicto & 6.01 & 1.81 & $.20^{* *}$ & $.48^{* *}$ & $-.41^{* *}$ & $-.25^{* *}$ & $.19^{* *}$ & - & & & & & & & \\
\hline 7. Cohesión & 6.76 & 2.22 & $-.21^{* *}$ & $-.42^{* *}$ & $.47^{* *}$ & $.29^{* *}$ & $-.24^{* *}$ & $-.51^{* *}$ & - & & & & & & \\
\hline 8. Organización & 6.23 & 1.81 & $-.14^{* *}$ & $-.27^{* *}$ & $.34^{* *}$ & $.21^{* *}$ & $-.13^{* *}$ & $-.36^{* *}$ & $.44^{* *}$ & - & & & & & \\
\hline \multicolumn{16}{|l|}{ Disciplina coercitiva } \\
\hline 9. Castigo & .59 & .53 & $.27^{* *}$ & $.58^{* *}$ & $-.36^{* *}$ & $-.17^{* *}$ & $.21^{* *}$ & $.49^{* *}$ & $-.43^{* *}$ & $-.22^{* *}$ & - & & & & \\
\hline 10. Coste de respuesta & .85 & .69 & $.10^{*}$ & $.27^{* *}$ & -.01 & .02 & $.12^{* *}$ & $.10^{*}$ & -.06 & -.00 & $.49^{* *}$ & - & & & \\
\hline 11. Supervisión & .65 & .65 & $.18^{* *}$ & $.42^{* *}$ & $-.21^{* *}$ & $-.13^{* *}$ & $.18^{* *}$ & $.22^{* *}$ & $-.24^{* *}$ & $-.15^{* *}$ & $.60^{* *}$ & $.56^{* *}$ & - & & \\
\hline \multicolumn{16}{|l|}{ Disciplina positiva } \\
\hline 12. Distracción & 1.12 & .93 & .05 & .06 & .01 & -.01 & $-.11^{*}$ & .03 & .00 & .00 & $.10^{*}$ & $.20^{* *}$ & .07 & - & \\
\hline 13. Explicación & 2.41 & 1.11 & .01 & .01 & .07 & .07 & -.04 & -.04 & $.10^{*}$ & .05 & -.02 & .05 & -.05 & $.29^{* *}$ & - \\
\hline 14. Recompensa & .72 & .74 & -.04 & .05 & .01 & -.02 & $-.11^{*}$ & -.04 & .04 & .02 & .05 & .09 & .03 & $.31^{* *}$ & $.30^{* *}$ \\
\hline
\end{tabular}

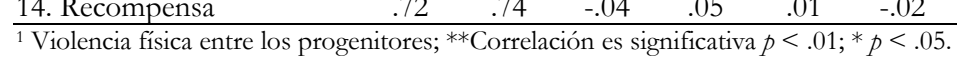




\section{Análisis Factorial Confirmatorio}

A través de un Análisis Factorial Confirmatorio (AFC) se comprobó la pertinencia del modelo de medida hipotetizado. Los índices de ajuste para el modelo AFC eran adecuados: $M L \chi^{2}(70, N=585)=216.06, C F I=.95, N N F I=.92$, $I F I=.95$, RMSE $A=.06$; Yuan-Bentler $\chi^{2}(70, N=585)=$ 200.71; CFI $=.93$, NNFI $=.90, I F I=.93$, RMSE $A=.057$. En el modelo se incluyó la correlación de errores entre la violencia marital (violencia física entre los padres) y violencia física (de hijos a padres) $(r=.40, p<.001)$. Todas las cargas factoriales resultaron significativas al nivel $p<.001$, excepto para la violencia física $(p<.01)$. De acuerdo con la Tabla 2, se obtuvieron correlaciones moderadas y elevadas entre Violencia Filio-Parental, Conducta Prosocial, Clima Familiar Positivo y Disciplina Coercitiva (todas $p<.001$ ). La variable violencia marital se asociaba significativamente con el resto de variables, aunque las correlaciones no eran elevadas. La condición de inmigrante se asociaba a un mayor nivel de violencia marital, disciplina coercitiva y a un menor nivel de conductas prosociales.

Tabla 2. Correlaciones entre las variables latentes y variables observables del modelo.

\begin{tabular}{|c|c|c|c|c|c|c|}
\hline & 1 & 2 & 3 & 4 & 5 & 6 \\
\hline \multicolumn{7}{|l|}{ Factores latentes } \\
\hline 1. Violencia Filio-Parental & -- & & & & & \\
\hline 2. Conducta Prosocial & $-.52 * *$ & -- & & & & \\
\hline 3. Clima Familiar Positivo & $-.68^{* *}$ & $.72 * *$ & -- & & & \\
\hline 4. Disciplina Coercitiva & $.70^{* *}$ & $-.40 * *$ & $-.59 * *$ & -- & & \\
\hline 5. Disciplina Positiva & .05 & .05 & .10 & .08 & -- & \\
\hline \multicolumn{7}{|l|}{ Variables observables } \\
\hline 6. Violencia marital & $.31 * *$ & $-.18^{* *}$ & $-.29 * *$ & $.27 * *$ & $-.17 *$ & -- \\
\hline 7. Inmigrante & .02 & .03 & $-.11 *$ & $.17^{*}$ & -.05 & $.20 * *$ \\
\hline
\end{tabular}

\section{Modelo estructural de la VFP}

El modelo estructural final se presenta en la Figura 1. El modelo muestra un ajuste razonable de los datos en base a la teoría normal: $M L \chi^{2}(82, N=585)=243.11 ; N N F I=.92$, $C F I=.94, I F I=.94$, RMSE $A=.058$. Sin embargo, con el método robusto de Yuan and Bentler (2000) la prueba chicuadrado escalada era 22 puntos inferior, aunque los índices de ajuste prácticos no mejoraron: $\mathrm{Y}-B \chi^{2}=220.26(\mathrm{RNNI}=$ $.91, \mathrm{RCFI}=.93, \mathrm{RIFI}=.93, \mathrm{RMSE} A=.054)$. Este modelo explicaba el $45 \%$ de la varianza de la violencia filio-parental.

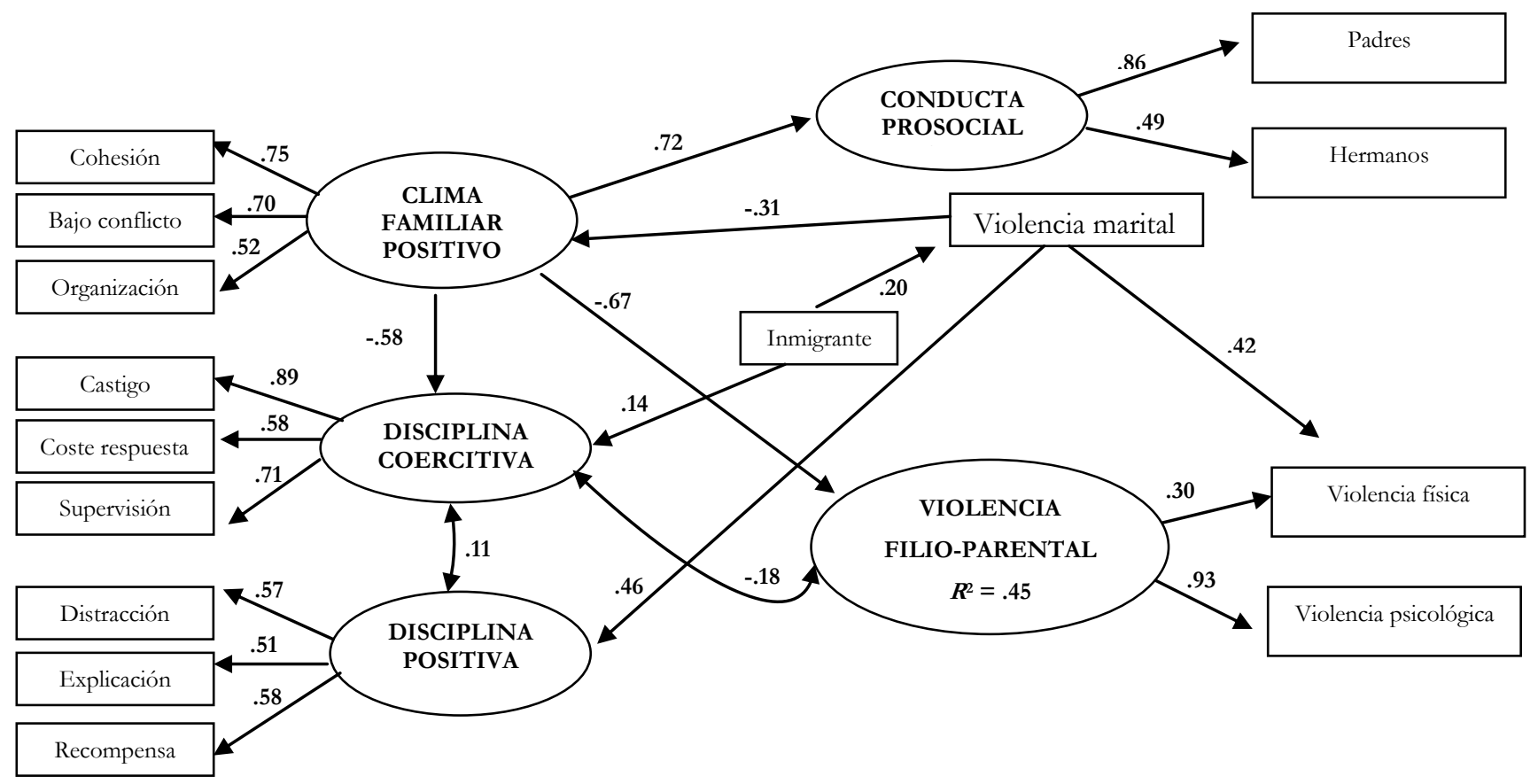

Figura 1. Modelo estructural de la violencia filio-parental. Todos los parámetros estimados son estandarizados. Todas las cargas factoriales, los coeficientes de regresión y correlaciones son significativos, $p<.01$. 
El Clima Familiar Positivo predecía un menor nivel de Violencia Filio-Parental $(\beta=-.67, p<.001)$ y Disciplina Coercitiva $(\beta=-.58, p<.001)$, así como un mayor nivel de Conducta Prosocial $(\beta=.72, p<.001)$. Además, la violencia marital era un predictor significativo de la violencia física de hijos a padres $(\beta=.42, p<.001)$, de un peor Clima Familiar $(\beta=-.31, p<.001)$ y de una menor utilización de la Disciplina Positiva $(\beta=-.18, p<.001)$. La condición de inmigrante estaba asociada significativamente a un mayor nivel de violencia marital $(\beta=.20, p<.001)$ y a una mayor utilización de estrategias de Disciplina Coercitiva por parte de los padres $(\beta=.14, p<.001)$. Además, la Disciplina Coercitiva covariaba moderadamente con la Violencia Filio-Parental $(r$ $=.46, p<.001)$, y en menor medida con la Disciplina Positiva $(r=.11, p<.05)$.

En la presente investigación el estudio de la relación entre la disciplina familiar y la violencia filio-parental es una cuestión interesante. Es posible que las estrategias de disciplina familiar de tipo coercitivo tengan un efecto negativo en las conductas violentas de los hijos, pero también podría ser que cuando los padres se encuentran ante conductas inadecuadas de los hijos adolescentes, éstos traten de reconducir la situación utilizando estrategias coercitivas. Por este motivo se probaron dos modelos alternativos: a) si la utilización de estrategias coercitivas por parte de los padres predice las conductas violentas de los hijos o b) si las conductas violentas de hijos a padres induce a la utilización de estrategias coercitivas. El ajuste del primer modelo era bueno, $M L \chi^{2}$ $(82, N=585)=248.36 ; N N F I=.92, C F I=.94, I F I=.94$, RMSE $A=.059 ; \mathrm{Y}-B \chi^{2}=225.12 ; \mathrm{RNNI}=.91, \mathrm{R} C F I=.93$, RIFI $=.93, \operatorname{RMSE} A=.055$, y la varianza explicada de la VFP era superior al modelo original $\left(R^{2}=.56\right)$. La Disciplina Coercitiva predecía positivamente la Violencia Filio-Parental $(\beta=.40, p<.001)$. El segundo modelo alternativo referido a la causalidad inversa tenía también buen ajuste, $M L \chi^{2}(82, N$ $=585)=239.49 ;$ NNFI $=.93$, CFI $=.94$, IFI $=.94$, RMSE $A=.057$; Y-B $\chi^{2}=216.58 ; \mathrm{RNNI}=.92, \mathrm{RCFI}=.93$, RIFI $=.93, \operatorname{RMSE} A=.053$, y la capacidad explicativa del modelo era similar $\left(R^{2}=.49\right)$. En este caso la Violencia Filio-Parental era también un predictor significativo de la Disciplina Coercitiva $(\beta=.58, p<.001)$.

\section{Discusión}

El objetivo de este estudio era analizar el papel del clima familiar y las estrategias de disciplina familiar según el nivel de coerción en las conductas violentas y prosociales de los adolescentes hacia sus padres y madres. Los resultados sugieren que el clima familiar positivo es un factor protector importante de la VFP mientras que la violencia marital física es un factor de riesgo. Por otro lado, todas las estrategias coercitivas de disciplina parental se asocian moderadamente a la violencia filio-parental mientras que las estrategias de disciplina no coercitiva (disciplina positiva) no se relacionan.
De acuerdo con la primera hipótesis, la violencia marital predecía significativamente la VFP física y coherente con este hallazgo el clima familiar positivo se asociaba a un menor nivel de conductas violentas de hijos a padres. Estos resultados están de acuerdo con la hipótesis de la bidireccionalidad de la violencia intra-familiar (Jaureguizar e Ibabe, 2012; Ibabe y Jaureguizar, 2011), por la cual los hijos que observan violencia entre los padres después reproducen este tipo de conductas contra sus padres y/o madres. Respecto al clima familiar se confirman los hallazgos de estudios previos que indican que los adolescentes agresores a diferencia de los no agresores tienen una visión más negativa del ambiente familiar y de las relaciones paternofiliares (Castañeda et al., 2012; Gallagher, 2008; Pagani et al., 2003). Parra y Oliva (2006) en un estudio longitudinal concluyeron que aquellos jóvenes que viven en un ambiente cálido y afectuoso tienen menos problemas conductuales. Dichos autores explican este hecho a través del mecanismo por el cual las buenas relaciones familiares aumentan el poder de influencia de los padres y madres sobre los hijos e hijas, disminuyendo la posible implicación del adolescente en conductas antisociales.

La segunda hipótesis se confirmó porque las estrategias coercitivas se asociaban positivamente a la violencia física de hijos a padres, y las estrategias de disciplina positiva no se asociaban con la violencia de hijos e hijas a padres. Los resultados de los modelos SEM alternativos inducen a pensar que la relación entre la disciplina coercitiva y la violencia filio-parental es bidireccional, en el sentido de que la aplicación de estrategias de disciplina coercitiva puede generar conductas violentas en los hijos, y viceversa, la incipiente aparición de problemas de conducta de los hijos, como señalan García-Linares, Cerezo, De la Torre, Carpio y Casanova (2011), determina la utilización de determinadas estrategias de disciplina por parte de los padres. Coherente con este hallazgo, Shek (2007) indicó una relación bidireccional entre el control de los padres y el bienestar de los hijos. Quizás por este motivo en el presente estudio, incluso la supervisión parental (estrategia de bajo nivel coercitivo) se asociaba positivamente con la violencia física y psicológica hacia los padres. Por otro lado, respecto a la disciplina inductiva Calvete et al. (2011) al igual que en el presente estudio, no hallaron ninguna asociación entre este tipo de disciplina y la violencia filioparental psicológica.

A falta de más estudios previos sobre violencia filioparental y disciplina familiar, se ha optado por comparar los resultados de este estudio con los hallazgos de los estudios centrados en la relación entre la disciplina familiar y el ajuste psicológico. No hay un consenso respecto a la influencia del control o disciplina familiar sobre el desarrollo del adolescente (García-Linares, De la Torre, Carpio, Cerezo y Casanova, 2014), porque a veces se ha constatado una falta de relación entre las estrategias de los progenitores para controlar el comportamiento adolescente y el ajuste psicológico del hijo, y otras veces una baja asociación inversa entre dichas variables (p.e., Kerr y Stattin, 2000). Durante décadas la inves- 
tigación científica ha mostrado cierto consenso en considerar que el control y el establecimiento de límites previenen la aparición de problemas conductuales (Fletcher, Steinberg y Williams-Wheeler, 2004). Así, en algunos estudios se ha encontrado que cuando los padres utilizan estilos de socialización basados en la baja coerción/imposición (escaso grado de exigencia), los hijos presentan más problemas de autocontrol (p.e., Lamborn et al., 1991) y de consumo de sustancias (Martínez, Fuertes, Ramos y Hernández, 2003). Los niveles de control conductual deberían estar ajustados a la edad y a las características del adolescente respetando al mismo tiempo el desarrollo autónomo para que influyan positivamente en su adaptación y bienestar (Parra y Oliva, 2006). Otros autores señalan que lo verdaderamente útil no es el nivel de control y supervisión parental, sino el hecho de que las negociaciones entre padres e hijos lleven a niveles de supervisión comúnmente aceptados (Martínez et al., 2003).

Las estrategias coercitivas y parcialmente coercitivas de disciplina parental se aplicaban con mayor frecuencia en las familias inmigrantes que en las familias autóctonas, confirmándose de esta manera la tercera hipótesis. Asimismo se observó un mayor nivel de violencia marital en estas familias. Los resultados sugieren que los padres y madres procedentes de contextos culturales diferentes al español aplican con más frecuencia estrategias coercitivas de disciplina familiar (castigo, coste de respuesta y supervisión) que los padres y madres autóctonos. En algunas culturas las prácticas de socialización jerarquizada y de firmeza parental se vinculan con el afecto positivo y el respeto por la autoridad (Martínez y García, 2008). A nivel internacional los estilos familiares democrático e indulgente se corresponden generalmente con mejores puntuaciones de ajuste personal de los hijos/as que los estilos autoritarios y negligentes. No obstante, en un reciente estudio a nivel nacional de García y García (2010) se encuentran indicios de que el estilo familiar idóneo en España es el indulgente (baja exigencia y elevado afecto), ya que las puntuaciones de los hijos de familias indulgentes siempre fueron equivalentes o incluso mejores, que las puntuaciones de las familias autorizativas (elevada exigencia y elevado afecto). Además, los resultados del estudio de GámezGuadix et al. (2012) sobre violencia filio-parental van en la misma línea, porque el estilo indulgente no se asociaba a una mayor probabilidad de informar de violencia filio-parental con población universitaria.

La cuarta hipótesis referida a la asociación entre la estructura familiar y las conductas violentas de los hijos e hijas se confirmó, ya que los adolescentes procedentes de familias nucleares presentaban más conductas violentas que las familias monoparentales con la madre (Kennair y Mellor, 2007), familias reconstituidas o familias de otro tipo. Pagani et al. (2003) hallaron que las familias intactas presentaban un menor nivel de violencia que las familias que habían sufrido algún cambio en su estructura. Esto podría ser explicado por ser las familias monoparentales y reconstituidas más vulnerables que las familias tradicionales en cuanto al clima familiar, por los eventuales conflictivos con su ex pareja o la adaptación a los nuevos miembros de la familia. Todo ello hace que en este tipo de familias haya un mayor nivel de estrés y escasez de recursos para afrontar la etapa adolescente del hijo/a. No obstante, las categorías familiares tratadas como entes globales no deben tener tal consideración por su mera composición (Antolín et al., 2009). Seguramente cuando la situación de monoparentalidad es originaria y no es derivada de un proceso de separación, la situación sea muy distinta. En esta investigación no se ha podido comprobar este dato, para ello se requeriría disponer de una muestra mucho más amplia o se podrían buscar perfiles concretos de estructura familiar. En cuanto a la violencia marital, contamos con evidencia empírica de que la población inmigrante española presenta mayores niveles de violencia de pareja y de creencias sexistas que la población española, lo cual puede ser explicado por la prevalencia y legitimación de la violencia en los países de origen (Arnoso, Ibabe, Arnoso y Elgorriaga, 2014).

La quinta hipótesis señalaba que las conductas prosociales hacia los padres se asociarían con el clima familiar positivo (Juareguizar e Ibabe, 2012; Romano et al., 2005) y con la disciplina inductiva. Esta hipótesis se confirmó en lo relacionado al clima familiar, ya que la cohesión, el bajo nivel de conflicto y organización aumentaban la probabilidad de dirigir conductas prosociales hacia los padres. Estos resultados están en la línea de lo hallado por Oliva, Parra y Arranz (2008) que destacan la importancia de la calidad de las relaciones entre padres e hijos para el desarrollo del adolescente. No obstante, la ausencia de relación entre las estrategias inductivas y las conductas violentas de hijos a padres, a diferencia de las estrategias coercitivas, se puede considerar un aspecto muy positivo. En la etapa de desarrollo que estamos analizando (12-18 años) los padres en numerosas ocasiones necesitarán utilizar estrategias de disciplina para ejercer de padres responsables, y las estrategias más recomendables serían las denominadas inductivas (explicación, distracción y recompensa).

En conclusión, las estrategias de disciplina familiar de alto y medio nivel coercitivo se relacionan con más violencia filio-parental, mientras que el clima familiar positivo predice menos violencia de este tipo y más conductas prosociales. Parece que en la sociedad española las relaciones familiares basadas en el afecto y la comunicación son las que favorecen las conductas prosociales de los hijos, y reducen las conductas violentas en el hogar. Un aspecto importante es la manera cómo los padres se dirigen a sus hijos y el tipo de relación que tengan con ellos. Cuando existe un clima emocional favorable entre padres e hijos, es más probable que los hijos se muestren receptivos a los intentos socializadores por parte de sus padres y no se revelen ante sus estrategias de control (Darling y Steinberg, 1993). Por el contrario, los padres percibidos como hostiles y distantes casi nunca tendrán una influencia positiva sobre los hijos, por lo que éstos podrían presentar conductas agresivas ante cualquier intento de los padres por controlar su comportamiento. Sin embargo, conviene recalcar que la supervisión parental es necesaria en to- 
dos los momentos de la vida de las hijas e hijos, pero se debe ajustar en cada momento a sus necesidades y madurez.

La principal limitación del presente estudio sería que, tal y como ocurre en las investigaciones transversales, no se puede establecer la dirección de la causalidad. El efecto marco podría influir en las respuestas de los participantes (Tversky y Kahneman, 1981): el hecho de que los participantes declaren que hay violencia familiar en su hogar, les podría inducir a indicar también la existencia de VFP, tratando de buscar consistencia en sus respuestas. Y, por otra parte, existe el riesgo de que la motivación de los participantes para responder pueda verse afectada por la deseabilidad social, por la cual podrían infravalorar la frecuencia de las conductas violentas o sobrevalorar la frecuencia de conductas prosociales en su familia por ser más aceptables socialmente. También es posible que algunas familias con elevado nivel

\section{Referencias}

Aluja, A., Del Barrio, V. y García, L. F. (2005). Relationships between adolescents' memory of parental rearing styles, social values and socialization behavior traits. Personality and Individual Differences, 39, 903-912. Doi:10.1016/j.paid.2005.02.028

Antolín, L., Oliva, A. y Arranz, E. (2009). Variables familiares asociadas a la conducta antisocial infantil: el papel desempeñado por el tipo de estructura familiar. Apuntes de Psicología, 27, 475-487.

Arbuckle, J. L. (1996). Full information estimation in the presence of incomplete data. En G. A. Marcoulides y R. E. Schumacker (Eds.), Advanced Structural Equation Modeling: Issues and Techniques (pp. 243-277). Mahwah, NJ: Lawrence Erlbaum Associates.

Arnoso, A., Ibabe, I., Arnoso, M. y Elgorriaga, E. (2014, noviembre). Las creencias sexistas como predictoras de la violencia en las relaciones de pareja en función del origen cultural. Comunicación presentada en XII Congreso Nacional de Psicología Social, Sevilla, España.

Baumrind, D. (1991). Parenting styles and adolescent development. En J. Brooks-Gun, R. Lerner y A. C. Petersen (Eds.), The encyclopedia of adolescence (pp. 746-758). New York: Garland.

Bentler, P. M. (2006). EQS, Structural Equations Program Manual. Encino, CA: Multivariate Statistical Software, Inc.

Beyers, W. y Goossens, L. (1999). Emotional autonomy, psychosocial adjustment and parenting: interactions, moderating and mediating effects. Journal of Adolescence, 22, 753-769. Doi:10.1006/jado.1999.0268

Bobic, N. (2004). Adolescent violence towards parents (pp.1-15). Sydney, New South Wales: Australian Domestic and Family Violence Clearing House.

Brezina, T. (1999). Teenage violence towards parents as an adaptation to family strain. Evidence from a National Survey of Male Adolescents. Youth and Society, 30, 416-444. Doi:10.1177/0044118X99030004002

Calvete, E., Gámez-Guadix, M. y Orue, I. (2010). El Inventario de Dimensiones de Disciplina (DDI), Versión niños y adolescentes: Estudio de las prácticas de disciplina parental desde una perspectiva de género. Anales de psicología, 26, 410-418.

Calvete, E., Orue, I. y Sampedro, R. (2011). Violencia filio-parental en la adolescencia: Características ambientales y personales. Infancia $y$ Aprendizaje, 34, 349-363. Doi: 10.1174/021037011797238577

Castañeda, A., Garrido-Fernández, M. y Lanzarote, M.D. (2011). Menores con conducta de maltrato hacia los progenitores: un estudio de personalidad y estilos de socialización. Revista de Psicología Social, 27, 157-167.

Chou, C. P. y Bentler, P. M. (1990). Model modification in covariance structure modeling: A comparison among likelihood ratio, Lagrange Multiplier, and Wald tests. Multivariate Behavioral Research, 25, 115-136.

Condry, R. (2011). Investigating adolescent violence towards parents. Respect Conference You just don't! Young people's violence in close. London, The Wellcome Collection Conference Centre, 17th February 2011. de conflicto nieguen el consentimiento informado para que participen sus hijos, lo cual supondría un sesgo de los resultados del estudio, al dejar fuera de la investigación los casos más graves de violencia filio-parental. Finalmente, en el presente estudio solamente se ha evaluado la violencia en el ámbito familiar a través de un informante (hijo/a), por lo que no es posible estudiar los comportamientos violentos en el contexto familiar desde distintas perspectivas. Aunque es conocida la existencia de discrepancias entre la información aportada por padres e hijos, esto no significa que los autoinformes de los adolescentes sean menos objetivos o fiables que los de sus padres.

Agradecimientos.- Esta investigación ha sido financiada por la dirección de Política Familiar y Comunitaria del Gobierno Vasco (M115/10).

Darling, N. y Steinberg, L. (1993). Parenting style as context: An integrative model. Psychological Bulletin, 113, 487-496. Doi: 10.1037//00332909.113.3.487

Fletcher, A. C., Steinberg, L. y Williams-Wheeler, M. (2004). Parental Influences on Adolescent Problem Behavior: Revisiting Stattin and Kerr. Child Development, 75, 781-796. Doi: 10.1111/i.1467-8624.2004.00706.x

Gallagher, E. (2008). Children's violence to parents: a critical literature review. Master thesis. Melbourne, Australia: Monash University. Recuperado de http://web.aanet.com.au/eddiegallagher/Violence $\% 20$ to $\% 20$ Parents $\%$ 20-\%20Gallagher $\% 202008$.pdf

Gámez-Guadix, M., Jaureguizar, J., Almendros, C. y Carrobles, J. A. (2012). Estilos de socialización familiar y violencia de hijos a padres en población española. Psicología Conductual, 20, 585-602.

Gámez-Guadix, M., Straus, M. A., Carrobles, J. A., Muñoz-Rivas, M. J. y Almendro, C. (2010). Corporal punishment and long-term behavior problems: The moderating role of positive parenting and psychological aggression. Psicothema, 22(4), 529-536.

García, F. y García, E. (2010). ¿Qué estilo de socialización parental es el idóneo en España? Un estudio con niños y adolescentes de 10 a 14 años. Infancia y Aprendizaje, 33, 365-384. Doi: 10.1174/021037010792215118

García-Linares, M. C., Cerezo, M.T., De la Torre, M.J., Carpio, M. V. y Casanova, P.F. (2011). Prácticas educativas paternas y problemas internalizantes y externalizantes en adolescentes españoles. Psicothema, 23, 654 659.

García-Linares, M. C., De la Torre, M. J., Carpio, M. V., Cerezo, M. T. y Casanova, P. F. (2014). Consistencia/inconsistencia en los estilos educativos de padres y madres, y estrés cotidiano en la adolescencia. Psicodidáctica. Preprint Doi://dx.doi.org/10.1387/RevPsicodidact.7219

Hetherington, E. M. y Henderson, S. H. (1997). Fathers in stepfamilies. En M. E. Lamb (Ed.), The role of the father in child development (pp. 212-226). Nueva York: Wiley and Sons.

Hetherington, E. M. y Stanley-Hagan, M.M. (2002). Parenting in divorced and remarried families. En M. H. Bornstein (Ed.), Handbook of Parenting (Vol. 3, pp. 233-254). Hove: LEA Publishers.

Holden, G. W. (2002). Perspectives on the effects of corporal punishment: Comment on Gershoff (2002). Psychological Bulletin, 128, 590-95. Doi: $\underline{10.1037 / / 0033-2909.128 .4 .590}$

Ibabe, I. y Jaureguizar, J. (2011). ¿Hasta qué punto la violencia filio-parental es bidireccional? Anales de Psicología, 27, 265-277.

Jaureguizar, J. e Ibabe, I. (2012). Conductas violentas de los adolescentes hacia las figuras de autoridad: el papel mediador de las conductas antisociales. Revista de Psicología Social, 27, 7-24. Doi: $\underline{10.1174 / 021347412798844088}$

Hu, L. T. y Bentler, P. M. (1999). Cutoff criteria for fit indexes in covariance structure analysis: conventional criteria versus new alternatives. Structural Equation Modeling, 6, 1-55. 
Kennair, N. y Mellor, D. (2007). Parent abuse: A review. Child Psychiatry and Human Development, 38, 203-219. Doi: 10.1007/s10578-007-0061-x

Kennedy, T. D., Edmonds, W. A., Dann, K. T. J. y Burnett, K. F. (2010). The clinical and adaptive features of young offenders of child-parent violence. Journal of Family Violence, 25, 509-520. Doi: 10.1007/s10896010-9312-x

Kerr, M. y Stattin, H. (2000). What Parents know, how they know it, and several forms of adolescent adjustment: Further support for a reinterpretation of monitoring. Developmental Psychology, 36, 366-380. Doi: $10.1037 / / 0012-1649.36 .3 .366$

Lamborn, S. D., Mounts, N. S., Steinberg, L. y Dornbusch, S. M. (1991). Patterns of competence and adjustment among adolescents from authoritative, authoritarian, indulgent, and neglectful families. Child Development, 62, 1.049-1.065.

Laurent, A. y Derry, A. (1999). Violence of French adolescents toward their parents. Journal of Adolescent Health, 25, 21-26.

Loeber, R. y Stouthamer-Loeber, M. (1986). Family factors as correlates and predictors of juvenile conduct problems and delinquency. En M. H. Tonry y N. Morris (Eds.), Crime and justice: An annual review of research, Vol. 7 (pp. 29-149). Chicago: University of Chicago Press. Doi: $\underline{10.1086 / 449112}$

Lorence, B. (2007). Procesos de socialización parental con adolescentes de familias en situación de riesgo psicosocial. Madrid: Fundación Acción Familiar.

Maccoby, E. E. y Martin, J. A. (1983). Socialization in the context of the family: Parent- child interaction. En P. H. Mussen (Ed.), Handbook of cbild psychology (Vol. 4, pp. 1-101). New York, NY: Wiley.

Martínez, I. y García, J. F. (2008). Internalization of values and self-esteem among Brazilian teenagers from authoritative, indulgent, authoritarian, and neglectful homes. Adolescence, 43, 13-29.

Martínez, J. L., Fuertes, A., Ramos, M. y Hernández, A. (2003). Consumo de drogas en la adolescencia: importancia del afecto y la supervisión parental. Psicothema, 15(2), 161-166. Recuperado de http://www.psicothema.com/pdf/1039.pdf

Matey, P. (2011, Octubre 6). Cuando los hijos maltratan a sus padres. El Mundo.

Recuperado http://www.elmundo.es/elmundosalud/2011/10/06/noticias/131792 6422.html

Mitchell, K. J. y Finkelhor, D. (2001). Risk of crime victimization among youth exposed to domestic violence. Journal of Interpersonal Violence, 16, 944-964. Doi: $10.1177 / 088626001016009006$

Moñivas, A. (1996). La conducta prosocial. Cuadernos de Trabajo Social, 9, 125142.

Moos, R. y Moos, B. (1981). Family Environment Scale. Manual. Palo Alto, CA: Consulting Psychologist Press.

Musitu, G. y García, J.F. (2004). Consecuencias de la socialización familiar en la cultura española. Psicothema, 16, 288-293. Recuperado de http://www.psicothema.com/pdf/1196.pdf

Musitu, G., Buelga, S., Lila, M. y Cava, M. J. (2001). Familia y adolescencia: un modelo de análisis e intervención psicosocial. Madrid: Editorial Síntesis, S.A.

Musitu, G., Román, J. M. y Gracia, E. (1988). Familia y Educación. Prácticas educativas de los padres y socialización de los hijos. Barcelona: Labor Universitaria.

No to Violence (2012). Adolescent Violence in the Home: Mapping the Australian and International Service System. Victoria, Australia. Recuperado de http://ntv.org.au/wp-content/uploads/121115-AVITH-report.pdf

Oliva, A. (2006). Relaciones familiares y desarrollo adolescente. Anuario de Psicología, 37, 209-223.

Oliva, A., Parra, A. y Arranz, E. (2008). Estilos relacionales parentales y ajuste adolescente. Infancia y Aprendiraje, 31, 93-106. Doi: $\underline{10.1174 / 021037008783487093}$

Pagani, L. S., Larocque, D., Vitaro, F. y Tremblay, R. E. (2003). Verbal and physical abuse toward mothers: The role of family configuration, environment, and doping strategies. Journal of Youth and Adolescence, 32, 215223.
Papalia, D. E., Olds, S. W. y Feldman, R. D. (2007). Human development $\left(10^{2}\right.$ ed.). Boston: McGraw-Hill.

Parra, A. y Oliva, A. (2006). Un análisis longitudinal sobre las dimensiones relevantes del estilo parental durante la adolescencia. Infancia y Aprendizaje, 29, 453-470. Doi: 10.1174/021037006778849594

Rodrigo, M.J., García, M., Márquez, M.L. y Triana, B. (2005). Discrepancias entre padres e hijos adolescentes en la frecuencia percibida e intensidad emocional en los conflictos familiares. Estudios de Psicología, 26, 21-34. Doi: $10.1174 / 0210939053421407$

Romano, E. Tremblay, R. E., Boulerice, B. y Swisher R. (2005). Multilevel correlates of childhood physical aggression and prosocial behavior. Journal of Abnormal Child Psychology, 33, 565-578. Doi: 10.1007/s10802005-6738-3

Shek, D. T. L. (2007). A longitudinal study of perceived differences in parental control and parental-child relational qualities in Chinese adolescents in Hong Kong. Journal of Adolescent Research, 22, 156-188.

Straus, M. y Stewart, J.H. (1999). Corporal punishment by American parents: National data on prevalence, chronicity, severity, and duration, in relation to child, and family characteristics. Clinical Child and Family Psychology Review, 2, 55-70.

Straus, M. A. y Douglas, E. (2004). A short form of the revised Conflict Tactics Scales, and typologies for severity and mutuality. Violence and Victims, 19, 507-520. Doi: $10.1891 / 088667004780927800$

Straus, M. A. y Fauchier, A. (2007). Manual for the Dimensions of Discipline Inventory (DDI). Durham, NH: Family Research Laboratory, University of New Hampshire.

Straus, M. A., Hamby, S., Boney-McCoy, S. y Sugarman, D. (1996). The Revised Conflict Tactics Scales Development and preliminary psychometric data. Journal of Family Issues, 17, 283-316. Doi: $\underline{10.1177 / 019251396017003001}$

Straus, M. A., Hamby, S.L., Finkelhor, D., Moore, D. W. y Runyan, D. (1998). Identification of child maltreatment with the parent-child conflict tactics scales: Development and psychometric data for a national sample of American parents. Cbild Abuse and Neglect, 22, 249-270. Doi: 10.1016/S0145-2134(97)00174-9

TEA Ediciones (1984). Escalas de clima social: familia, trabajo, instituciones penitenciarias, centro escolar. Madrid: TEA.

Tversky, A. y Kahneman, D. (1981). The framing of decisions and the psychology of choice. Science, 211, 453-458.

Ulman, A. y Straus, M. A. (2003). Violence by children against mothers in relation to violence between parents and corporal punishment by parents. Journal of Comparative Family Studies, 34, 41-60.

Walsh, J. A. y Krienert, J. L. (2007). Child-parent violence: An empirical analysis of offender, victim, and event characteristics in a national sample of reported incidents. Journal of Family Violence, 22, 563-574. Doi: 10.1007/s10896-007-9108-9

Weiss, L. H. y Schwarz, J. C. (1996). The relationship between parenting types and older adolescents' personality, academic achievement, adjustment, and substance use. Child Development, 67, 2101-2114. Doi: $10.2307 / 1131612$

Werner, E. (1989). High risk children in young adulthood: A longitudinal study from birth to 32 years. American Journal of Orthopsychiatry, 59, 59-71. Doi: j.1939-0025.1989.tb01636.x

Yoshikawa, H. (1994). Prevention as cumulative protection: Effects of early family support and education on chronic delinquency and its risks. Psychological Bulletin, 115, 28-54. Doi: 10.1037//0033-2909.115.1.28

Yuan, K. H, Lambert, P. L. y Fouladi, R. T. (2004). Mardia's multivariate kurtosis with missing data. Multivariate Behavioral Research, 3, 413 - 437. Doi: dx.doi.org/10.1207/S15327906MBR3903 2

Yuan, K. H. y Bentler, P.M. (2000). Three likelihood-based methods for mean and covariance structure analysis with nonnormal missing data. Sociological Methodology, 30, 165-200.Doi: dx.doi.org/10.1111/00811750.00078

(Articulo recibido: 17-5-2013; revisado: 17-01-2014; aceptado: 12-03-2014) 